Check for updates

Cite this: RSC Adv., 2019, 9, 11670

\title{
Preparation of thin solid electrolyte by hot- pressing and diamond wire slicing
}

\author{
Masashi Kotobuki, (D) ${ }^{\text {ab }}$ Houhua Lei, ${ }^{c}$ Yu Chen, (D) bd Shufeng Song, (D) e \\ Chaohe Xu, (D) ${ }^{\text {e Ning Hu}}{ }^{e}$ Janina Molenda (iD ${ }^{f}$ and $\mathrm{Li} \mathrm{Lu}^{* a b}$
}

The thickness of a solid electrolyte influences the performance of all-solid-state batteries due to increased impedance with a thick electrolyte. Thin solid electrolytes are favourable to improve the performance of all-solid-state batteries due to the short Li ion diffusion path and small volume of the solid electrolytes. Therefore, the preparation of thin solid electrolyte is one of the key process techniques for development of all-solid-state batteries. In this study, thin $\mathrm{Li}_{1.5} \mathrm{Ge}_{1.5} \mathrm{Al}_{0.5}\left(\mathrm{PO}_{4}\right)_{3}$ solid electrolyte with a $\mathrm{Na}$ super ion conductor structure is prepared by diamond wire slicing. The $\mathrm{Li}_{1.5} \mathrm{Ge}_{1.5} \mathrm{Al}_{0.5}\left(\mathrm{PO}_{4}\right)_{3}$ solid electrolyte is prepared by melt-quenching followed by crystallization at $800^{\circ} \mathrm{C}$ for $8 \mathrm{~h}$, after which the crystallized $\mathrm{Li}_{1.5} \mathrm{Ge}_{1.5} \mathrm{Al}_{0.5}\left(\mathrm{PO}_{4}\right)_{3}$ rod is subjected to wire slicing. Thin $\mathrm{Li}_{1.5} \mathrm{Ge}_{1.5} \mathrm{Al}_{0.5}\left(\mathrm{PO}_{4}\right)_{3}$ with a thickness of $200 \mu \mathrm{m}$ is obtained. The crystal structure and cross-sectional morphology are not affected by the slicing. The total $\mathrm{Li}$ conductivity of the thin $\mathrm{Li}_{1.5} \mathrm{Ge}_{1.5} \mathrm{Al}_{0.5}\left(\mathrm{PO}_{4}\right)_{3}$ and activation energy are $3.3 \times 10^{-4} \mathrm{~S} \mathrm{~cm}^{-1}$ and $0.32 \mathrm{eV}$, respectively. The thickness and total conductivity are comparable to those of $\mathrm{Li}_{1.5} \mathrm{Ge}_{1.5} \mathrm{Al}_{0.5}\left(\mathrm{PO}_{4}\right)_{3}$ prepared by the tape-casting method which needs several steps to prepare $\mathrm{Li}_{1.5} \mathrm{Ge}_{1.5} \mathrm{Al}_{0.5}\left(\mathrm{PO}_{4}\right)_{3}$ tape-sheet and high temperature and a long sintering process. The ionic transference number of the thin $\mathrm{Li}_{1.5} \mathrm{Ge}_{1.5} \mathrm{Al}_{0.5}\left(\mathrm{PO}_{4}\right)_{3}$ is 0.999. The diamond wire slicing is a useful method to prepare thin solid electrolytes.

Received 26th January 2019
Accepted 5th April 2019

DOI: 10.1039/c9ra00711c

rsc.li/rsc-advances
In an all-solid-state battery, the thickness of the solid electrolyte influences the performance as schematically illustrated in Fig. 1. The purpose of an electrolyte in a battery is to prevent short circuit between the positive and negative electrodes but allow $\mathrm{Li}$ ions to transfer from the anode to the cathode during discharge and vice versa. Since Li ion migration between the two electrodes is a diffusion process, a thick solid electrolyte will lead to an increase in impedance of the all-solid-state battery and hence difficulties of charge transfer. Additionally, the electrolyte is an inactive material, and a large volume of the thick electrolyte implies a lower volumetric and gravimetric power and energy densities. ${ }^{17}$ Therefore, process techniques to prepare thin and dense solid electrolyte are essential.

For preparation of thin solid electrolytes, physical vapour processes such as sputtering and PLD (pulse laser (a)

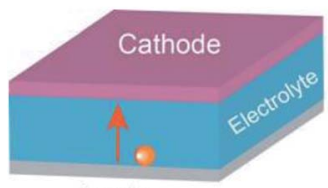

Anode

High cell resistance Low power density Low energy density (b)

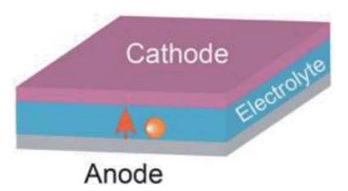

Low cell resistance High power density High energy density
Fig. 1 Performances of all-solid-state batteries with (a) thick and (b) thin solid electrolytes. 
deposition $)^{\mathbf{1 8 , 1 9}}$ and liquid processes like the sol-gel method ${ }^{\mathbf{2 0}}$ have been studied. However, these physical processes have some disadvantages such as usage of vacuum system, difficulty in control of chemical composition and large area coating. Also, in both processes, the thin solid electrolytes must be deposited on a substrate and it is difficult to obtain self-standing thin electrolytes. Although a simple method to obtain the selfstanding thin solid electrolytes is to grind a thick solid electrolyte pellet to a thin one, this process is time-consuming and easy to create defect. Imanishi et al. applied the tape-casting method for preparation of thin LAGP solid electrolyte ${ }^{21}$ and obtained thin LAGP with a thickness of $228 \mu \mathrm{m}$. Using this processing, a total conductivity $3.38 \times 10^{-4} \mathrm{~S} \mathrm{~cm}^{-1}$ was achieved. $^{22}$ This verified the tape-casting method was useful for thin solid electrolyte preparation, however, the tape-casting method has a number of drawback, including complexity of processing and composition deviation from desired one.

To obtain the self-standing thin electrolytes, the simple slicing technique using a diamond saw is considered to be useful and was applied for the garnet-type solid electrolytes, ${ }^{23,24}$ however, the diamond saw slicing tends to form kerf and waste materials. Contrary, the diamond wire slicing using a wire impregnated with diamond dust produces less kerf and wasted materials compared with the diamond saw slicing. Additionally, the diamond wire slicing is also practical and less expensive process. $^{25}$

In this study, the diamond wire slicing was applied for LAGP solid electrolyte first time. By the diamond wire slicing of thick LAGP pellet, thin LAGP slices of a thickness of about $200 \mu \mathrm{m}$ can be successfully prepared.

\section{Experimental}

\subsection{Preparation of LAGP solid electrolyte}

In order to slice the LAGP solid electrolyte, thick LAGP rod with a length of about $2 \mathrm{~cm}$ was needed. In a typical processing, the LAGP rods were prepared by melt-quenching method followed by hot-press technique. ${ }^{26}$ The precursors of $\mathrm{Li}_{2} \mathrm{CO}_{3}(>99.99 \%)$, $\mathrm{GeO}_{2}(>99.999 \%), \mathrm{Al}_{2} \mathrm{O}_{3}$ (99.98\%) and $\mathrm{NH}_{4} \mathrm{H}_{2} \mathrm{PO}_{4}$ (>98\%) which were all purchased from Sigma Aldrich were mixed in ethanol, dried and heated at $380{ }^{\circ} \mathrm{C}$ for $2 \mathrm{~h}$ in an $\mathrm{Al}$ crucible. To compensate Li evaporation, $10 \mathrm{~mol} \%$ excess $\mathrm{Li}_{2} \mathrm{CO}_{3}$ was added. After grinding, the obtained powder was melted in a Pt crucible at $1350{ }^{\circ} \mathrm{C}$ for $2 \mathrm{~h}$ and casted on a stainless plate pre-heated at $500{ }^{\circ} \mathrm{C}$. After solidification, the cast glass was annealed at $500{ }^{\circ} \mathrm{C}$ for $2 \mathrm{~h}$ to release the thermal stress. The glass was crushed into powder. The crushed glass powder was loaded into a graphite die of $10 \mathrm{~mm}$ in a diameter and then subjected to hot-press sintering. ${ }^{27}$ Since glass transition temperature, $T_{\mathrm{g}}$, and crystallization temperature, $T_{\mathrm{c}}$, are 530 and $603{ }^{\circ} \mathrm{C}$, respectively, ${ }^{27}$ to fully utilize rubber-like behaviour, the glass powder was hotpressed at $600{ }^{\circ} \mathrm{C}$ under a pressure of $20 \mathrm{MPa}$ for $1 \mathrm{~h}$ in an $\mathrm{Ar}$ atmosphere. After hot-press sintering, the sintered-glass was crystallized at $800{ }^{\circ} \mathrm{C}$ for $8 \mathrm{~h}$ in a heating rate of $3{ }^{\circ} \mathrm{C} \mathrm{min}$ min $^{-1}$ in and under atmospheric pressure to obtain crystallized LAGP. This batch sample is denoted as sample 1. For a comparison, the hot-press sintered-glass without crystallization was also prepared. This batch of samples was crystallized in same condition after slicing and denoted as sample 2 as shown in Fig. 2.

\subsection{Slicing LAGP solid electrolyte}

As a tradition processing, a diamond wire was used to slice the rod. To avoid heat effect, aqueous lubricant coolant was used during whole slicing process. The slicing rate was adjusted to about $0.1 \mathrm{~mm} \mathrm{~min}^{-1}$.

\subsection{Characterization}

As a crystal structure of the thin LAGP was identified by XRD measurement (XRD-6000, Shimadzu) with $\mathrm{Cu} \mathrm{K}_{\alpha}$ radiation from $2 \theta=10$ to $60^{\circ}$ with a step of $0.02^{\circ}$ at a scan rate of $1^{\circ} \mathrm{min}^{-1}$. Microstructure and morphology of the thin LAGP solid electrolytes was observed by scanning electron microscope (SEM, JEOL-6010 PLUS/LV) operating at $15 \mathrm{kV}$. Au was sputtered on the samples to add an electronic conductivity and eliminate the charge effect. Densities of the thin LAGP solid electrolytes were measured by using a pycnometer (AccuPyc II 1340, Micromeritics).

The Li ion conductivity of the thin LAGP was estimated by the electrochemical impedance spectroscopy method. Prior to measurement, $\mathrm{Au}$ was sputtered on both surfaces of the samples to prepare the $\mathrm{Li}$ ion blocking electrodes and ensure electrical contact. The impedance measurement was performed at a voltage signal of $10 \mathrm{mV}$ in a frequency range of $10-1 \mathrm{MHz}$ at 28-150 ${ }^{\circ} \mathrm{C}$ using a Solartron impedance analyser $1470 \mathrm{E}$ cell test system. The activation energy was calculated from Arrhenius plot using following equation.

$$
\sigma_{\mathrm{t}}=A \exp \left(-E_{\mathrm{a}} / k_{\mathrm{B}} T\right)
$$

where $\sigma_{\mathrm{t}}, E_{\mathrm{a}}, k_{\mathrm{B}}, A$ and $T$ are total Li ion conductivity, activation energy, Boltzmann constant, pre-exponential factor and absolute temperature, respectively.

Ionic transference number of the thin LAGP solid electrolyte was measured by DC polarization technique. ${ }^{28}$ A DC voltage of $1.0 \mathrm{~V}$ was applied to the Au/LAGP/Au cell. The ionic transference number $\left(t_{\mathrm{i}}\right)$ was calculated from initial current, $I_{\mathrm{i}}$ and stabilized current, $I_{\mathrm{f}}$, using following equation:

$$
t_{\mathrm{i}}=\frac{I_{\mathrm{i}}-I_{\mathrm{f}}}{I_{\mathrm{i}}}
$$

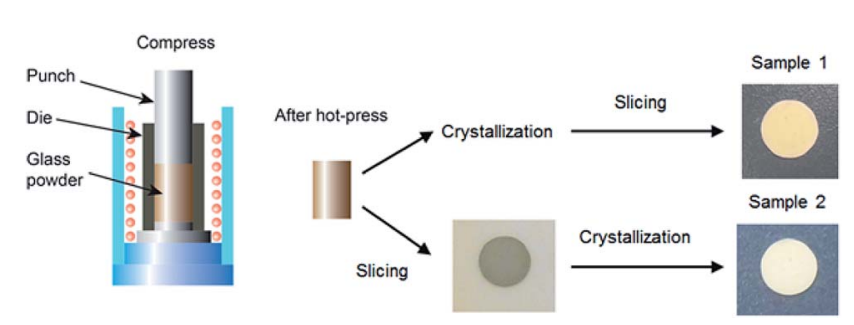

Fig. 2 A scheme of preparation of thin LAGP by the diamond wire slicing. 


\section{Results and discussion}

\subsection{Characterization of thin LAGP solid electrolyte}

Fig. 3 shows images of the thin LAGP pellets. For the sample 1, the white coloured thin pellet with $200 \mu \mathrm{m}$ in a thickness was obtained. In the diamond saw slicing of garnet-type electrolytes, thicknesses of pellets were almost same $(0.18-0.22 \mathrm{~cm}) \cdot{ }^{23,24}$ For the sample 2, the thin pellet with grey colour was changed into white colour through the crystallization. The thickness of the sample 2 before the crystallization was $290 \mu \mathrm{m}$. The thickness became slightly smaller due to shrinkage during the crystallization, while diameter of the sample 2 did not change through the crystallization.

XRD patterns of the thin LAGP pellets were depicted in Fig. 4a. In both samples, main phase could be attributed to LAGP with NASICON structure. Also, small peaks of $\mathrm{GeO}_{2}$ and $\mathrm{Al}_{6} \mathrm{Ge}_{2} \mathrm{O}_{13}$ was also observed in both diffraction patterns. Table 1 reveals lattice parameters of the sample 1 and 2 . For a comparison, LAGP solid electrolyte crystallized at same temperature without the slicing is also shown in Table $1 .^{26}$ The sample 1 possessed slightly larger lattice parameter in a-axis than that of the sample without slicing, whereas c-axis of the sample 1 was much shorter. For the sample 2, a-axis was shorter than that of others, while c-axis of the sample 2 was a little longer than the sample 1 and much shorter than the sample without slicing. As a result, the lattice volume of the sample 1 was larger than that of the sample 2 and smaller than that of the sample without slicing. This observation implied that the sample preparation history influenced lattice parameters. The influence of the sample history on the properties of samples was shown more clearly in morphological observation.

Fig. $4 \mathrm{~b}$ shows cross-sectional SEM images of the sample 1 and 2. For the sample 1 (Fig. $4 \mathrm{~b}(1)$ and (2)), many densely packed small crystal grains whose sizes were about $2 \mu \mathrm{m}$, were observed. No porosity was noted. These morphological features were very similar to the sample without slicing. ${ }^{26}$ In contrary, the sample 2 (Fig. $4 \mathrm{~b}(3)$ and (4)) showed different crosssectional morphologies. Crystal grains showed non-uniform size ranging from $100 \mathrm{~nm}$ to $>10 \mu \mathrm{m}$.

Additionally, there were some pores in the cross-section. It was also noted that there was a large difference in the relative density. The relative densities of the sample 1 and 2 are 98.9 and $88.7 \%$, respectively. We studied history of the processing and
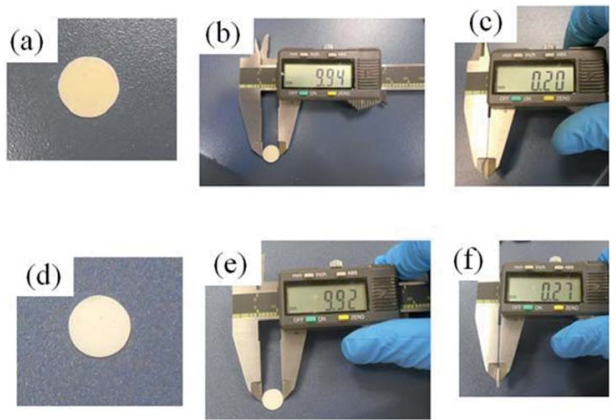

Fig. 3 Photos and dimensions of $(a-c)$ sample 1 and $(d-f)$ sample 2 (a)
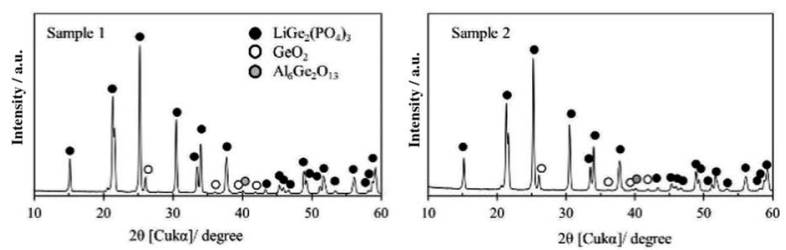

(b)
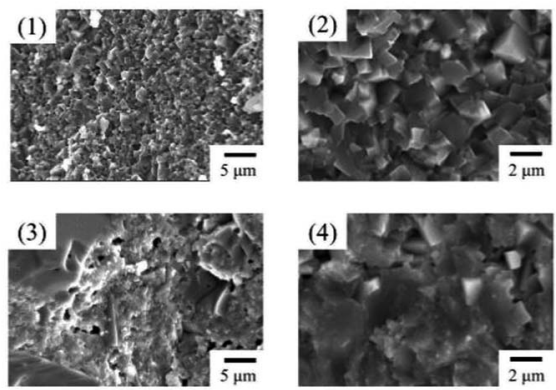

Fig. 4 (a) XRD patterns of sample 1 and sample 2, (b) cross-sectional SEM images of ( 1 and 2 ) sample 1 and ( 3 and 4 ) sample 2.

noted that the hot-pressing was carried out at the temperature between $T_{\mathrm{g}}$ and $T_{\mathrm{c}}$ where the glass powder showed a rubber-like behaviour. Therefore, it is easy to achieve a very dense pellet even at $20 \mathrm{MPa}$ as illustrated in Fig. 5(a). However, although the glass powder could be hot-pressed to almost no porosity, powder particles were roughly bonded mechanically since low pressing temperature and short pressing duration would be unable to achieve sufficient chemical bonding. A good chemical bonding was achieved at $800{ }^{\circ} \mathrm{C}$ during the crystallization (Fig. 5(b1)). Since a good bonding was obtained in the crystallization process, dense thin slices with no or little damage could be obtained (Fig. 5(c1)). However, if the slicing was carried out directly after hot-pressing, cleavage crack and impact from the diamond wire could cause delamination and crack as shown in Fig. 5(b2). The delamination and cracks would be further expanded due to shrinkage during post-crystallization process (Fig. 5(c2)).

\subsection{Electrochemical properties of thin LAGP solid electrolyte}

The Li ion conductivity of the thin LAGP samples was evaluated by the electrochemical impedance method. Fig. 6 depicts impedance spectra of the sample 1 and 2 measured at $28{ }^{\circ} \mathrm{C}$. In both spectra, a semicircle and a tail were observed in the highand low-frequency range, respectively. This characteristic profile of the impedance plot often appears in ceramics that are

Table 1 Lattice parameters of thin LAGP prepared by mechanical slicing $^{a}$

\begin{tabular}{llll}
\hline Sample & $a(\AA)( \pm 0.002)$ & $c(\AA)( \pm 0.002)$ & $V\left(\AA^{3}\right)( \pm 0.4)$ \\
\hline Thick LAGP & 8.262 & 20.656 & 1221.0 \\
Sample 1 & 8.265 & 20.619 & 1219.8 \\
Sample 2 & 8.255 & 20.624 & 1217.0
\end{tabular}

${ }^{a}$ Ref. 26. 


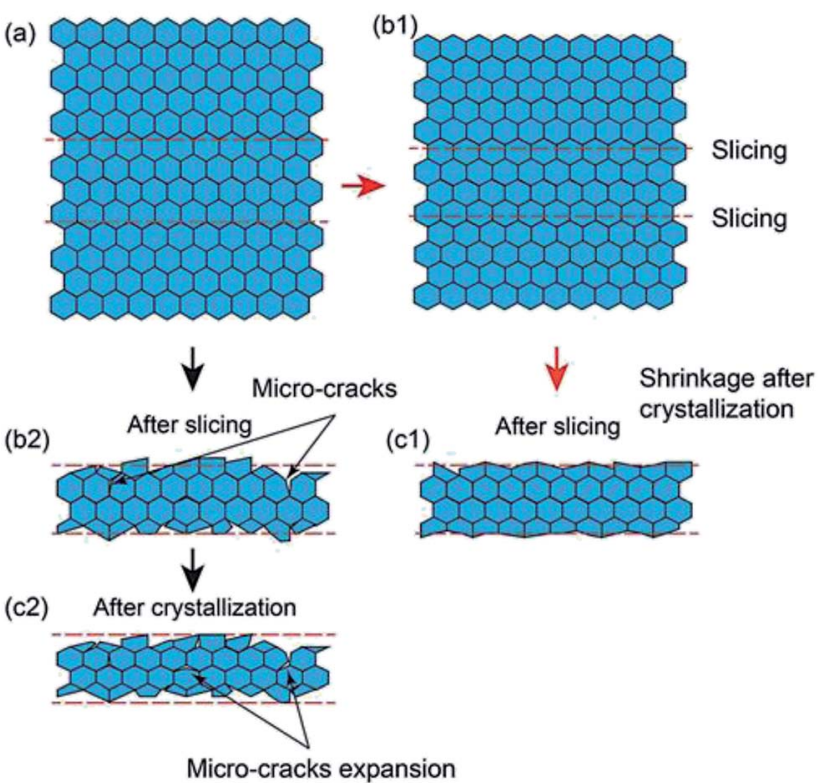

Fig. 5 Schematic illustration on difference of sample morphologies in the history of processing

ion conductive..$^{29,30}$ The tail corresponds to capacitive blocking behaviour of the electrodes. ${ }^{31}$

The intercepts of the semicircle at the high- and lowfrequency sides are assigned to inner crystal and total (inner crystal and grain boundary) impedances, respectively. Estimated bulk (inner crystal) and total conductivities of the sample 1 were $5.9 \times 10^{-4}$ and $3.3 \times 10^{-4} \mathrm{~S} \mathrm{~cm}^{-1}$, respectively. Imanishi et al. prepared thin LAGP with a thickness of $228 \mu \mathrm{m}$ and reported its total conductivity was $3.38 \times 10^{-4} \mathrm{~S} \mathrm{~cm}^{-1} .22$ Although the thickness and total conductivity were comparable to those of the sample 1 , the diamond wire slicing can simplify the
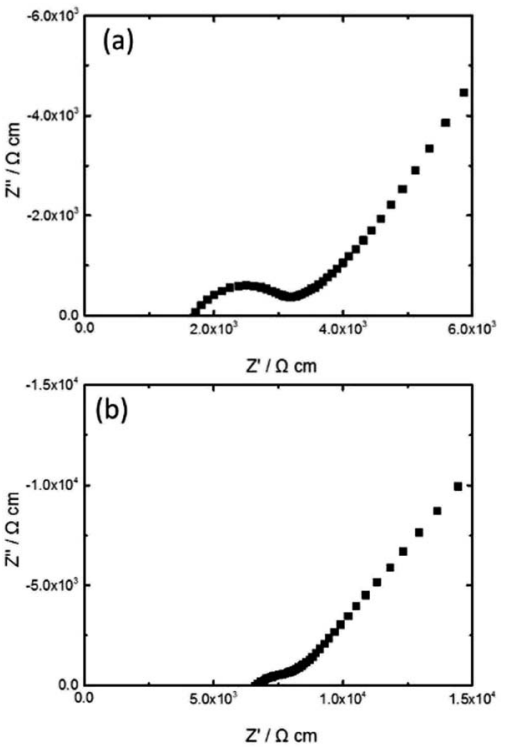

Fig. 6 Complex impedance plots of (a) sample 1 and (b) sample 2 measured at $28{ }^{\circ} \mathrm{C}$ in a voltage signal of $10 \mathrm{mV}$. preparation process significantly. Fig. 7 reveals a comparison experimental procedures of tape-casting and mechanical slicing methods. The tape-casting method requires many steps including twice long ball-milling processes. Contrary, this diamond wire slicing needs only 4 steps to obtain thin LAGP. This simple technique can reduce not only time and energy for thin LAGP preparation, but also production cost. In the sample 2, a depressed semicircle was observed. This would be because grain boundaries were non-uniform due to uneven growth of crystal grains. The bulk and total conductivities of the sample 2 were $1.5 \times 10^{-4}$ and $1.2 \times 10^{-4} \mathrm{~S} \mathrm{~cm}^{-1}$, respectively. Both conductivities were lower than those of the sample 1 due to pores, non-uniform crystal grains and poor bonding.

Based on temperature dependence of the Li ion conductivities, activation energy of the sample 1 was calculated. Above $75{ }^{\circ} \mathrm{C}$, the bulk and total impedances could not be separated due to a disappearance of the semicircle. Therefore, only total conductivity was used for the activation energy calculation. The Arrhenius plot (Fig. 8a) could be fitted by a straight line and hence the activation energy was calculated to be $0.32 \mathrm{eV}$ from slope of the straight line. This value is same as that of tapecasting LAGP $(0.32 \mathrm{eV}) .{ }^{22}$

Ionic transference number of the sample 1 was examined using Au/LAGP/Au cell by the DC polarization technique. The polarization curve is shown in Fig. 8b. The ionic transference number can be calculated by the initial and stabilized current. The calculated ionic transference number is $>0.999$, indicating that the sample 1 is a pure ionic conductor.

It is verified that the diamond wire slicing is a very simple and useful method to obtain thin solid electrolytes. In this study, the hot-press was used to prepare thick LAGP rod, however, conventional sintering method also can be used if thick rod could be obtained. Additionally, this slicing technique can be applied for other solid electrolytes such as garnet- and perovskite-type solid electrolytes. Furthermore, by optimization of slicing condition, thinner solid electrolyte would be obtained. These attempts are being studied in our group. The results will be reported in due course.
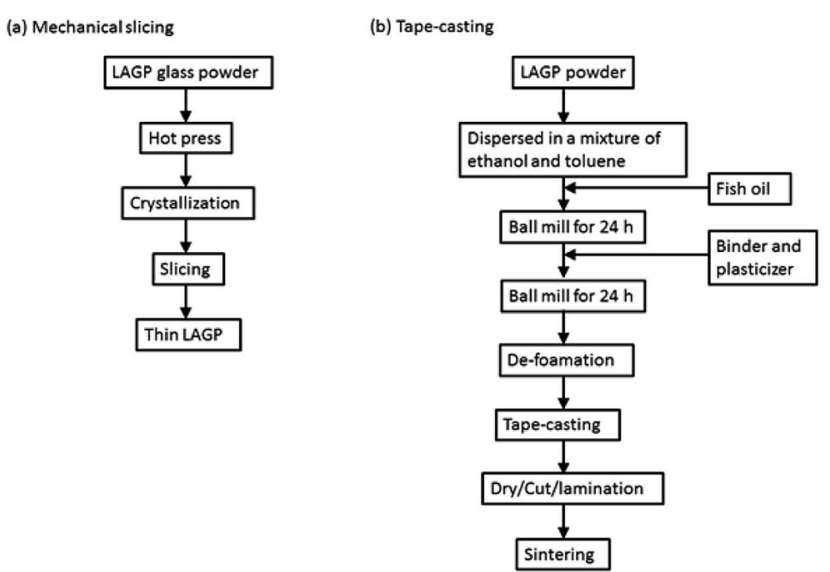

Fig. 7 A comparison of experimental procedures of (a) diamond wire slicing and (b) tape-casting methods. ${ }^{22}$ 

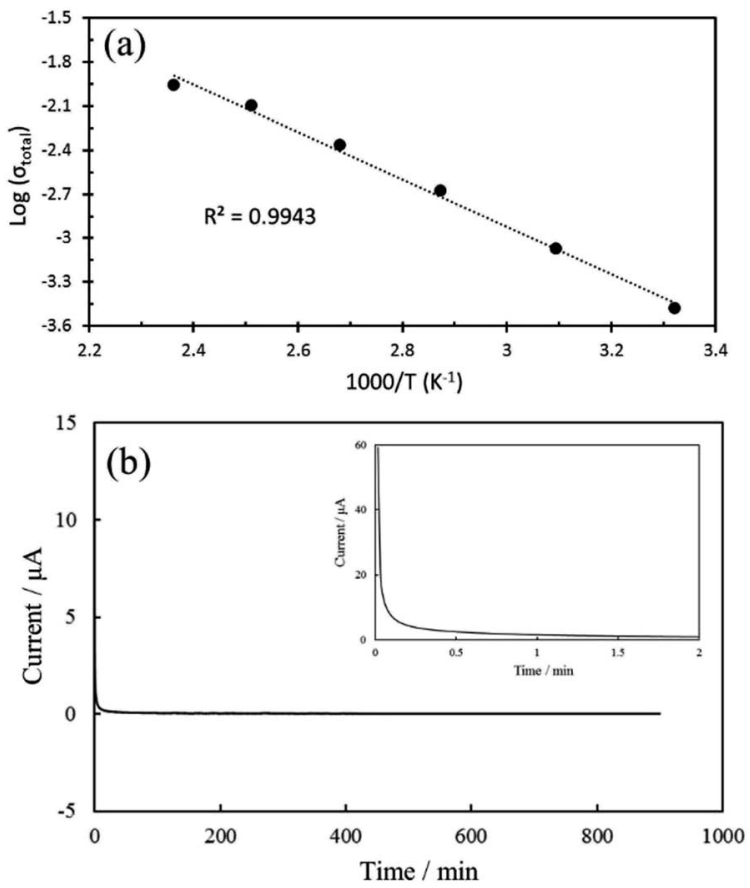

Fig. 8 (a) Arrhenius plot of total Li ion conductivity of sample 1 and (b) chronoamperometric curve of the sample 1 measured at an applied voltage of $1 \mathrm{~V}$

\section{Conclusions}

Thin LAGP solid electrolyte was prepared by hot-pressing and simple diamond wire cutting. Two kinds of samples were fabricated for the cutting: one was crystallized LAGP and another one was glass LAGP. The glass LAGP was crystallized after the slicing. Both samples revealed very similar XRD patterns in which main phase was LAGP with NASICON structure, while morphology was very different. The sample sliced after the crystallization showed uniformly grown crystal grains with about $2 \mu \mathrm{m}$ in size and no gap among the crystal grains. In contrary, the sample crystallized after the slicing exhibited non-uniform crystal growth. Sizes of crystal grains were a range from $100 \mathrm{~nm}$ to $>10 \mu \mathrm{m}$. Additionally, there were some pores in the cross-section. As a result, the sample sliced after crystallization revealed higher bulk and total $\mathrm{Li}$ ion conductivities, $5.9 \times 10^{-4}$ and $3.3 \times 10^{-4} \mathrm{~S} \mathrm{~cm}^{-1}$ in a thickness of $200 \mu \mathrm{m}$, respectively. The thickness and $\mathrm{Li}$ ion conductivity were comparable to LAGP prepared by the tape-casting method which needs several steps to prepare LAGP tape-sheet and high temperature and long sintering process. The diamond wire slicing is a simple and useful method to obtain thin solid electrolytes. Thinner solid electrolyte would be obtained by optimization of slicing conditions.

\section{Conflicts of interest}

There are no conflicts to declare.

\section{Acknowledgements}

This work is supported by National University of Singapore, National University (Suzhou) Research Institute, Solid-Force Pte
Ltd (Suzhou, China), the National Natural Science Foundation of China (NSFC 51572182, 11502036, 11372104, 11632004), the Natural Science Fund of the city of Chongqing (cstc2015jcyjA0577), and the Key Program for International Science and Technology Cooperation Projects of the Ministry of Science and Technology of China (No. 2016YFE0125900).

\section{Notes and references}

1 Z. Feng, M. Kotobuki, S. Song, M. O. Lai and L. Lu, J. Power Sources, 2018, 389, 198-213.

2 K. Hoshina, K. Yoshima, M. Kotobuki and K. Kanamura, Solid State Ionics, 2012, 209-210, 30-35.

3 M. Kotobuki, Y. Isshiki, H. Munakata and K. Kanamura, Electrochim. Acta, 2010, 55, 6892-6896.

4 A. A. Raskovalov, E. A. Il'ina and B. D. Antonov, J. Power Sources, 2013, 238, 48-52.

5 S. Song, Z. Dong, F. Deng and N. Hu, Funct. Mater. Lett., 2018, 11, 1850039.

6 M. Kotobuki and M. Koishi, Ceram. Int., 2014, 40, 5043-5047.

7 M. Kotobuki, S. Song, R. Takahashi, S. Yanagiya and L. Lu, J. Power Sources, 2017, 349, 105-110.

8 S. Qin, X. Zhu, Y. Jiang, M. Ling, Z. Hu and J. Zhu, Funct. Mater. Lett., 2018, 11, 1850029.

9 M. Kotobuki, Y. Suzuki, K. Kanamura, Y. Sato, K. Yamamoto and T. Yoshida, J. Power Sources, 2011, 196, 9815-9819.

10 S. Stramare, V. Thangadurai and W. Weppner, Chem. Mater., 2003, 15, 3974-3999.

11 J. Wu, L. Chen, T. Song, Z. Zou, J. Gao, W. Zhang and S. Shi, Funct. Mater. Lett., 2017, 10, 1730002.

12 M. Kotobuki and M. Koishi, Ceram. Int., 2013, 39, 4645-4649.

13 M. Kotobuki, M. Koishi and Y. Kato, Ionics, 2013, 19, 19451948.

14 J. K. Feng, L. Lu and M. O. Lai, J. Alloys Compd., 2010, 501, 255-258.

15 M. Kotobuki and M. Koishi, Ceram. Int., 2015, 41, 8562-8567. 16 M. Kotobuki, E. Hanc, B. Yan, J. Molenda and L. Lu, Ceram. Int., 2017, 43, 12616-12622.

17 Ceramic electrolytes for all-solid-state Li batteries, ed. M. Kotobuki, S. Song, C. Chen and L. Lu, World Scientific Publishing Co. Pte. Ltd., 2018.

18 B. J. Hwang, C. Y. wang, M. Y. Cheng and R. Santhanam, J. Phys. Chem. C, 2009, 113, 11373-11380.

19 N. Kuwata, R. Kumar, K. Toribami, T. Suzuki, T. Hattori and J. Kawamura, Solid State Ionics, 2006, 177, 2827-2832.

20 K. Tadanaga, H. Egawa, A. Hayashi, M. Tatsumisago, J. Mosa, M. Aparicio and A. Duran, J. Power Sources, 2015, 273, 844-847.

21 M. Zhang, K. Yakahashi, I. Uechi, Y. Takeda, O. Yamamoto, D. Im, D.-J. Lee, B. Chi, J. Pu, J. Li and N. Imanishi, J. Power Sources, 2013, 235, 117-121.

22 M. Zhang, Z. Huang, J. Cheng, O. Yamamoto, N. Imanishi, B. Chi, J. Pu and J. Li, J. Alloys Compd., 2014, 590, 147-152.

23 V. Thangadurai and W. Weppner, J. Solid State Chem., 2006, 179, 974-984.

24 R. Murugan, V. Thangadurai and W. Weppner, Angew. Chem., Int. Ed., 2007, 46, 7778-7781. 
25 https://en.wikipedia.org/wiki/Wire_saw.

26 Y. Zhu, Y. Zhang and L. Lu, J. Power Sources, 2015, 290, 123129.

27 B. Yan, Y. Zhu, F. Pan, J. Liu and L. Lu, Solid State Ionics, 2015, 278, 65-68.

28 M. Kotobuki, L. Lu, S. V. Savilov and S. M. Aldoshin, J. Electrochem. Soc., 2017, 164(14), A3868-A3875.
29 V. Thangadura and W. Weppener, J. Power Sources, 2005, 142, 339-344.

30 M. Kotobuki, K. Kanamura, Y. Sato, K. Yamamoto and T. Yoshida, J. Power Sources, 2012, 190, 346-349.

31 A. Kubanska, L. Castro, L. Tortet, O. Schaef, M. Dolle and R. Bouchet, Solid State Ionics, 2014, 266, 44-50. 